01

\title{
Релятивистские расчеты химических свойств сверхтяжелого элемента с $Z=119$ и его гомологов
}

\author{
(ㄱ И.И. Тупицын, А.В. Малышев, Д.А. Глазов, М.Ю. Кайгородов, Ю.С. Кожедуб, И.М. Савельев, В.М. Шабаев \\ Санкт-Петербургский государственный университет, \\ 199034 Санкт-Петербург, Россия \\ e-mail: i.tupitsyn@spbu.ru
}

Поступила в редакцию 20.03.2021 г.

В окончательной редакции 20.03.2021 г.

Принята к публикации 30.03.2021 г.

В рамках методов конфигурационного взаимодействия и многочастичной теории возмущений $(\mathrm{CI}+\mathrm{MBPT})$ в базисе орбиталей Дирака-Фока-Штурма (DFS) выполнены релятивистские расчеты электронной структуры сверхтяжелого элемента восьмого периода - эка-франция $(Z=119)$ и его гомологов, которые образуют группу щелочных металлов. Полученные значения потенциалов ионизации, сродства к электрону и среднеквадратичных радиусов сравниваются с соответствующими величинами, рассчитанными в нерелятивистском приближении. Проведено также сравнение с доступными экспериментальными данными и результатами других теоретических расчетов. Анализ полученных результатов свидетельствует о значительном влиянии релятивистских эффектов для атомов франция и эка-франция, приводящем к нарушению монотонного характера перечисленных выше химических характеристик как функции атомного номера щелочного элемента. Кроме того, вычислены квантово-электродинамические поправки к потенциалам ионизации с использованием модельного оператора лэмбовского сдвига (QEDMOD).

Ключевые слова: сверхтяжелые элементы, щелочные металлы, релятивистские эффекты, корреляционные эффекты, квантово-электродинамические поправки, метод наложения конфигураций, потенциалы ионизации, сродство к электрону.

DOI: $10.21883 /$ OS.2021.07.51074.2033-21

\section{1. Введение}

Синтез и изучение сверхтяжелых ядер представляют собой одну из важнейших задач ядерной физики [1-3]. Физические и химические свойства соответствующих сверхтяжелых элементов (СТЭ) представляют как прикладной, так и фундаментальный интерес. С одной стороны, сам процесс детектирования атомов СТЭ тесно связан с их способностью вступать в соединения. С другой стороны, вопрос о том, в какой степени для СТЭ сохраняются свойства более легких гомологов, по сути определяет границы применимости периодического закона Менделеева. При этом экспериментальное исследование свойств СТЭ в настоящее время затруднено в виду крайне малого количества синтезируемых атомов и их очень короткого времени жизни. Таким образом, теоретические данные о свойствах СТЭ очень востребованы. Очевидно, что такие расчеты наряду с электронными корреляциями должны учитывать релятивистские и квантово-электродинамические (КЭД) эффекты. Электронная структура СТЭ интенсивно исследуется несколькими научными группами [4-12].

В данной работе представлены расчеты химических характеристик эка-франция $(Z=119)$ и его более легких гомологов, образующих первую группу таблицы Менделеева, группу щелочных металлов. Ранее в разных работах (например, [13] и ссылки в ней) было установлено, что основной конфигурацией эка-франция является
$[\mathrm{Rn}] 5 f^{14} 6 d^{10} 7 s^{2} 7 p^{6} 8 s^{1}$. Наиболее точные релятивистские расчеты электронной структуры эка-франция и его гомологов были выполнены методом связанных кластеров [14-18]. В настоящей работе мы используем релятивистские методы конфигурационного взаимодействия и многочастичной теории возмущений (CI+MBPT) в базисе орбиталей Дирака-Фока-Штурма (DFS). Вклады релятивистских эффектов оценены посредством сравнения результатов релятивистских расчетов с данными, которые получены теми же методами в нерелятивистском пределе путем масштабирования скорости света. В рамках метода модельного оператора лэмбовского сдвига также вычислен вклад КЭД эффектов. Показано, что, несмотря на значительный вклад релятивистских эффектов, свойства эка-франция соответствуют первой группе таблицы Менделеева.

\section{2. Теория. Краткое описание метода расчета}

В данной работе мы используем релятивистский гамильтониан Дирака-Кулона-Брейта (Dirac-CoulombBreit — DCB) $[19,20]$

$$
\hat{H}_{\mathrm{DCB}}=\Lambda_{+}\left[\hat{H}_{\mathrm{D}}+\hat{H}_{\mathrm{C}}+\hat{H}_{\mathrm{B}}\right] \Lambda_{+} \cdot
$$


$\hat{H}_{\text {D }}$ - сумма одноэлектронных четырехкомпонентных гамильтонианов Дирака,

$$
\begin{gathered}
\hat{H}_{\mathrm{D}}=\sum_{i}^{N} \hat{h}_{\mathrm{D}}(i), \\
\hat{h}_{\mathrm{D}}(i)=-i c\left(\boldsymbol{\alpha}_{i} \cdot \nabla_{i}\right)+c^{2}\left(\beta_{i}-1\right)+V_{\mathrm{n}}\left(r_{i}\right),
\end{gathered}
$$

где $N$ - число электронов, $c$ - скорость света, $\boldsymbol{\alpha}$ и $\beta$ - матрицы Дирака, $V_{\mathrm{n}}(r)-$ потенциал ядра. Здесь и далее в работе используются атомные единицы. Для описания распределения заряда ядра мы применяем модель Ферми с параметром толщины оболочки равным $t=2.3 \mathrm{fm}$. Значение среднеквадратичного радиуса ядра для эка-франция рассчитано по эмпирической формуле $\left\langle r^{2}\right\rangle^{1 / 2}=\left[0.77 A^{1 / 3}+0.98\right] \mathrm{fm}$, где $A-$ атомное число, которое для данного элемента принято равным 295. Для гомологов значения среднеквадратичных радиусов ядер взяты из табуляции [21]. Оператор $\hat{H}_{\mathrm{C}}-$ двухэлектронный оператор кулоновского взаимодействия,

$$
\hat{H}_{\mathrm{C}}=\frac{1}{2} \sum_{i \neq j}^{N} \frac{1}{r_{i j}}, \quad r_{i j}=\left|\mathbf{r}_{i}-\mathbf{r}_{j}\right| .
$$

Оператор $\hat{H}_{\mathrm{B}}-$ двухэлектронный частотно-независимый оператор Брейта в кулоновской калибровке

$\hat{H}_{\mathrm{B}}=-\frac{1}{2} \sum_{i \neq j}^{N} \frac{1}{r_{i j}}\left[\left(\boldsymbol{\alpha}_{i} \cdot \boldsymbol{\alpha}_{j}\right)+\frac{1}{2}\left(\boldsymbol{\alpha}_{i} \cdot \nabla_{i}\right)\left(\boldsymbol{\alpha}_{j} \cdot \nabla_{j}\right) r_{i j}\right]$.

Оператор $\Lambda_{+}$проектирует многоэлектронные волновые функции на пространство детерминантов Слэтера, построенных из одноэлектронных состояний положительного спектра.

\section{1. Метод Дирака-Фока-Штурма}

Многоэлектронная волновая функция $\Psi(J M)$ с квантовыми числами $J$ и $M$ в приближении DCB может быть получена в виде разложения по конфигурационным функциям (Configuration State Functions - CSF) $\Phi_{I}(J M)$ :

$$
\Psi(J M)=\sum_{I} C_{I}^{J M} \Phi_{I}(J M)
$$

где $\Phi_{I}(J M)$ являются собственными функциями операторов $\hat{J}^{2}$ и $\hat{J}_{z}$ и строятся как линейные комбинации детерминантов Слэтера одной релятивистской конфигурации.

Вариационный принцип Ритца в пространстве CSF сводит решение уравнения DCB к задаче на собственные значения матрицы гамильтониана:

$$
\sum_{K} H_{K I} C_{K}^{J M}=E_{I}(J) C_{I}^{J M}
$$

где $H_{K I}=\left\langle\Phi_{K}\left|\hat{H}_{\mathrm{DCB}}\right| \Phi_{I}\right\rangle$, а индексы $I$ и $K$ нумеруют различные CSF.
В настоящей работе одноэлектронные волновые функции $\psi_{i}$ получены методом Дирака-Фока-Рутана в базисе орбиталей Дирака-Фока-Штурма (Dirac-Fock-Sturm DFS) $\varphi_{k}$ :

$$
\psi_{i}=\sum_{k} u_{k i} \varphi_{k}
$$

В свою очередь, базис DFS построен следующим образом. В качестве орбиталей $\varphi_{k}$, которые заняты в основном и низколежащих возбужденных состояниях, использованы одноэлектронные волновые функции, полученные численным решением интегро-дифференциальных уравнений Дирака-Фока (Dirac-Fock - DF) [22]. Для виртуальных (высоколежащих вакантных) одноэлектронных состояний функции $\varphi_{k}$ получены путем численного решения уравнений Дирака-Фока-Штурма [22,24],

$$
\left[\hat{h}_{\mathrm{DF}}-\varepsilon_{0}\right] \varphi_{k}=\mu_{k} W(r) \varphi_{k}
$$

где $\hat{h}_{\mathrm{DF}}$ - оператор Дирака-Фока, $\varepsilon_{0}-$ ссылочная одноэлектронная энергия и $W(r)$ - положительная весовая функция, стремящаяся к нулю на бесконечности. Отметим, что все орбитали DFS имеют примерно одинаковый характерный размер и одну и ту же асимптотику на бесконечности, определяемую ссылочной энергией $\varepsilon_{0}$ :

$$
\varphi_{k}(r) \underset{r \rightarrow \infty}{\longrightarrow} C_{k} \exp \left(-\sqrt{2 \varepsilon_{0}} r\right)
$$

В качестве весовой функции $W(r)$ выбрана функция, стремящаяся к константе при $r \rightarrow 0$ :

$$
W(r)=\frac{1-\exp \left(-(\alpha r)^{2}\right)}{(\alpha r)^{2}} .
$$

В данной работе во всех расчетах использован единый базис одноэлектронных функций $\psi_{i}$.

\section{2. Метод наложения конфигурации в сочетании с многочастичной теорией возмущений (Cl+MBPT)}

Для учета электронных корреляций в данной работе применяется объединение методов конфигурационного взаимодействия (Configuration Interaction - CI) и многочастичной теории возмущений (Many-Body Perturbation Theory - MBРТ). Для построения конфигурационного пространства использована концепция ограниченного активного пространства (Restricted Active Space RAS) [25] в базисе орбиталей DFS [23,24]. Согласно этому подходу, набор одноэлектронных функций разбивается на 4 подгруппы: RAS0, RAS1, RAS2 и RAS3.

K подпространству RAS0 отнесены так называемые замороженные остовные орбитали, возбуждения из которых не учитываются. Электроны замороженного остова создают одночастичные кулоновский и обменный потенциалы остова и не участвуют в CI+MBPT расчете. В подпространство RAS1 включены орбитали внешнего 
остова, однократные и двукратные возбуждения из которого учитываются по теории возмущений. Активные валентные орбитали отнесены к подпространству RAS2, а виртуальные высоковозбужденные орбитали - к RAS3. Однократные и двукратные возбуждения из RAS1 и RAS2 в данной работе учтены по теории возмущений во втором порядке. В валентном пространстве RAS2 строится CI-функция путем решения задачи на собственные значения матрицы гамильтониана (7).

Теория возмущений хорошо подходит для учета большого числа возбуждений, а также обладает важным свойством размерной согласованности. Метод конфигурационного взаимодействия позволяет рассматривать возбуждения в активном пространстве во всех порядках. Используемый нами подход гибко и эффективно использует преимущества обоих методов, что становится особенно важно с ростом числа электронов.

\section{3. Квантово-электродинамические поправки}

Для учета КЭД эффектов в данной работе использован одноэлектронный модельный оператор лэмбовского сдвига $\hat{h}^{\mathrm{QED}}$ (QEDMOD), который был предложен в работе [26] и широко применяется в различных квантово-химических расчетах [27-32]. Квантовоэлектродинамическая поправка вычислена как разность двух полных энергий, рассчитанных методом СІ. В одном из расчетов модельный КЭД оператор включен в гамильтониан DCB, тогда как другой расчет проведен без модельного оператора. Модельный оператор $\hat{h}^{\mathrm{QED}}$ состоит из двух частей,

$$
\hat{h}^{\mathrm{QED}}=\hat{h}^{\mathrm{VP}}+\hat{h}^{\mathrm{SE}} \text {. }
$$

Операторы $\hat{h}^{\mathrm{VP}}$ и $\hat{h}^{\mathrm{SE}}$ представляют вклады поляризации вакуума и собственной энергии соответственно. Оператор $\hat{h}^{\mathrm{VP}}$ может быть представлен в виде суммы локальных потенциалов Юлинга $V_{\mathrm{Uehl}}(r)$ и ВичманнаКролла $V_{\mathrm{WK}}(r)$ :

$$
\hat{h}^{\mathrm{VP}}=V_{\mathrm{Uehl}}(r)+V_{\mathrm{WK}}(r) .
$$

Потенциала Юлинга представляет собой ведущий вклад по степеням взаимодействия с ядром [33,34]. Он может быть получен как численно, так и аналитически с помощью приближенных формул из работы [35]. Вычисление потенциала Вичманна-Кролла представляет существенно более сложную задачу [36-38]. Однако вклад данного потенциала можно оценить с достаточно высокой точностью по приближенным формулам, представленным в статье [39].

Оператор собственной энергии $\hat{h}^{\mathrm{SE}}$, описание которого подробно изложено в работе [26], моделируется в виде суммы локального короткодействующего потенциала $V_{\text {loc }}^{\mathrm{SE}}$ и нелокального сепарабельного (конечномерного) потенциала

$$
\hat{h}^{\mathrm{SE}}=V_{\mathrm{loc}}^{\mathrm{SE}}+\sum_{i, k=1}^{n}\left|\phi_{i}\right\rangle B_{i k}\left\langle\phi_{k}\right| .
$$

Таблица 1. Среднеквадратичные радиусы (RMS) и среднеквадратичные отклонения (STD) валентной $n s$-оболочки (в а.u.). DF - релятивистский метод Дирака-Фока, DF-NR нерелятивистский предел

\begin{tabular}{r|c|c|c|c}
\hline \multirow{2}{*}{$Z$} & \multicolumn{2}{|c|}{ RMS } & \multicolumn{2}{c}{ STD } \\
\cline { 2 - 5 } & DF-NR & DF & DF-NR & DF \\
\hline 11 & 4.55 & 4.54 & 1.73 & 1.73 \\
19 & 5.62 & 5.60 & 2.01 & 2.01 \\
37 & 6.01 & 5.93 & 2.11 & 2.09 \\
55 & 6.71 & 6.48 & 2.29 & 2.23 \\
87 & 7.04 & 6.31 & 2.37 & 2.17 \\
119 & 7.54 & 5.54 & 2.49 & 1.91
\end{tabular}

Здесь $\phi_{i}-$ специальным образом выбранные проекционные функции, а матрица $B_{i k}$ определяется так, чтобы в точности воспроизвести известные диагональные и недиагональные матричные элементы однопетлевого оператора собственной энергии в базисе водородоподобных функций [26]. Компьютерный код для генерации оператора QEDMOD представлен в работах [40,41].

Наиболее простым подходом к вычислению КЭД поправок является усреднение модельного оператора $\hat{h}^{\mathrm{QED}}$ с многоэлектронными волновыми функциями. В наших расчетах мы с самого начала включаем модельный оператор $\hat{h}^{\mathrm{QED}}$ в гамильтониан DCB. Такой подход дополнительно учитывает высшие порядки по модельному потенциалу, которые могут давать существенный вклад [32].

\section{3. Результаты и обсуждение}

В данной работе расчеты эка-франция $(Z=119)$ и его гомологов выполнены релятивистскими методами DF и CI+MBPT в базисе орбиталей Дирака-Фока-Штурма. С целью изучения изменения химических характеристик при переходе от легких щелочных металлов к сверхтяжелым нами рассчитаны потенциалы ионизации, значения сродства к электрону, а также характерные размеры валентной $n s$-оболочки.

В табл. 1 приведены релятивистские и нерелятивистские значения среднеквадратичных радиусов (RootMean-Square radius - $\left.\mathrm{RMS}=\bar{r}=\left\langle n s\left|r^{2}\right| n s\right\rangle^{1 / 2}\right)$ и среднеквадратичных отклонений (Standard Deviation $\left.\mathrm{STD}=\left\langle n s\left|(r-\bar{r})^{2}\right| n s\right\rangle^{1 / 2}\right)$, которые характеризуют размеры атомов щелочных металлов и ширину распределения электронной плотности валентной $n s$-оболочки соответственно. Кроме того, эти же данные представлены на рис. 1,2. Отметим, что величина STD есть мера локализации валентных $n s$-оболочек. Релятивистские значения RMS и STD получены методом ДиракаФока, их нерелятивистские аналоги рассчитаны путем увеличения скорости света в 100 раз. Из таблицы и графиков хорошо видно, что нерелятивистские значения 
Таблица 2. Потенциалы ионизации (IP) элементов первой группы таблицы Менделеева (в eV). DF - метод ДиракаФока, CI+MBPТ - метод наложения конфигураций в сочетании с теорией возмущений; NR - нерелятвистский предел

\begin{tabular}{r|c|c|c|c}
\hline \multicolumn{1}{c|}{$Z$} & DF-NR & DF & CI+MBPT-NR & CI+MBPT \\
\hline 11 & 4.961 & 4.961 & 5.151 & 5.159 \\
19 & 4.012 & 4.026 & 4.303 & 4.323 \\
37 & 3.808 & 3.808 & 4.097 & 4.177 \\
55 & 3.365 & 3.486 & 3.723 & 3.889 \\
87 & 3.208 & 3.603 & 3.578 & 4.079 \\
119 & 2.995 & 4.327 & 3.258 & 4.780
\end{tabular}

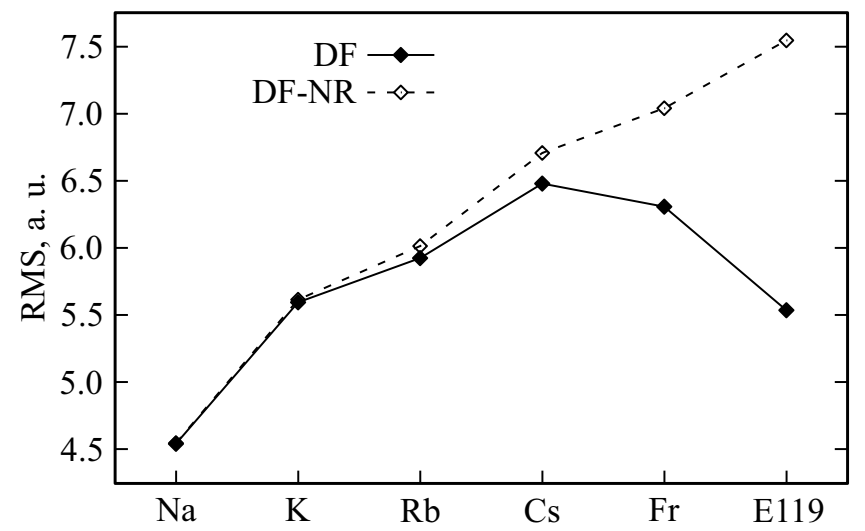

Рис. 1. Среднеквадратичные радиусы (RMS) валентной $n s$ оболочки (в а.u.). DF - релятивистский метод Дирака-Фока, DF-NR - нерелятивистский предел.

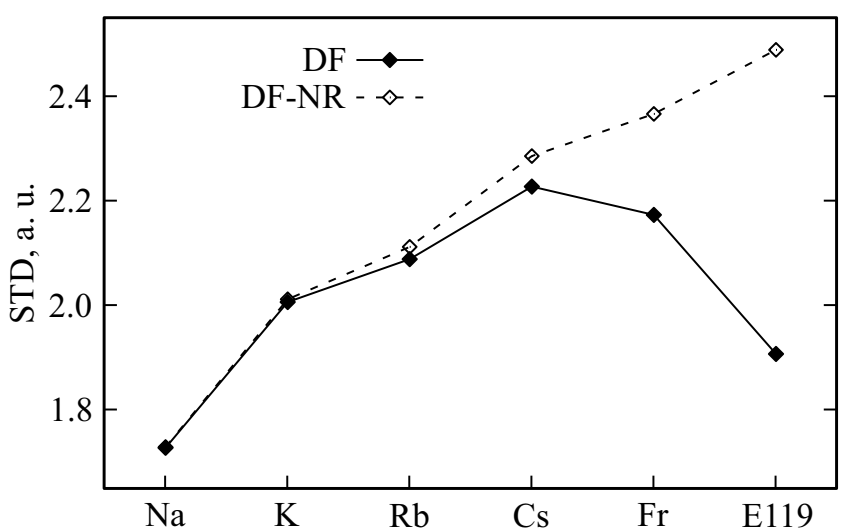

Рис. 2. Среднеквадратичные отклонения (STD) для валентной $n s$-оболочки (в а.u.). Обозначения такие же, как на рис. 1.

RMS и STD монотонно возрастают с ростом $Z$, что свидетельствует о делокализации валентных $n s$-состояний. Однако, как видно из этих же рисунков, учет релятивистских эффектов приводит к нарушению монотонного характера изменений значений RMS и STD, начиная с $Z=87$. Это обстоятельство связано с контрактацией $n s$ орбиталей и увеличением степени их локализации, что является чисто релятивистским эффектом.
В табл. 2 и 3 приведены значения потенциалов ионизации (Ionization Potential - IP) и сродства к электрону (Electron Affinity - EA) эка-франция и его более легких гомологов, рассчитанные релятивистскими методами DF и CI+MBPT, а также их значения в нерелятивистском пределе. Для всех элементов использован базис, который состоит из орбиталей Дирака-Фока для занятых в основном состоянии оболочек атома и иона, а также набора виртуальных орбиталей Дирака-ФокаШтурма. Базис виртуальных орбиталей включает в себя $7 s, 7 p, 7 d, 5 f, 4 g$ и $3 h$ штурмовских функций. При вычислении потенциалов ионизации мы используем единый базис, построенный для нейтрального атома. В расчетах сродства к электрону использован базис, построенный для отрицательного иона.

Как видно из табл. 2 и 3 и графиков на рис. 3 и 4, значения потенциалов ионизации и сродства к электрону, рассчитанные в нерелятивистском пределе методами DF и CI+MBPT, монотонно спадают с ростом атомного номера. Такое поведение значений IP и ЕА коррелирует с ростом степени делокализации нерелятивистских значений RMS и STD. Учет релятивистских эффектов нарушает монотонный характер поведения значений IP и EA, так же как и для значений RMS и STD, начиная с $Z=87$.

Таблица 3. Значения сродства к электрону (EA) для элементов первой группы таблицы Менделеева (в $\mathrm{eV})$. Обозначения такие же, как в табл. 2

\begin{tabular}{r|c|c|c|c}
\hline \multicolumn{1}{c|}{$Z$} & DF-NR & DF & CI+MBPT-NR & CI+MBPT \\
\hline 11 & 0.363 & 0.364 & 0.551 & 0.553 \\
19 & 0.281 & 0.282 & 0.504 & 0.505 \\
37 & 0.259 & 0.264 & 0.473 & 0.488 \\
55 & 0.227 & 0.237 & 0.453 & 0.475 \\
87 & 0.214 & 0.251 & 0.431 & 0.498 \\
119 & 0.197 & 0.388 & 0.423 & 0.674
\end{tabular}

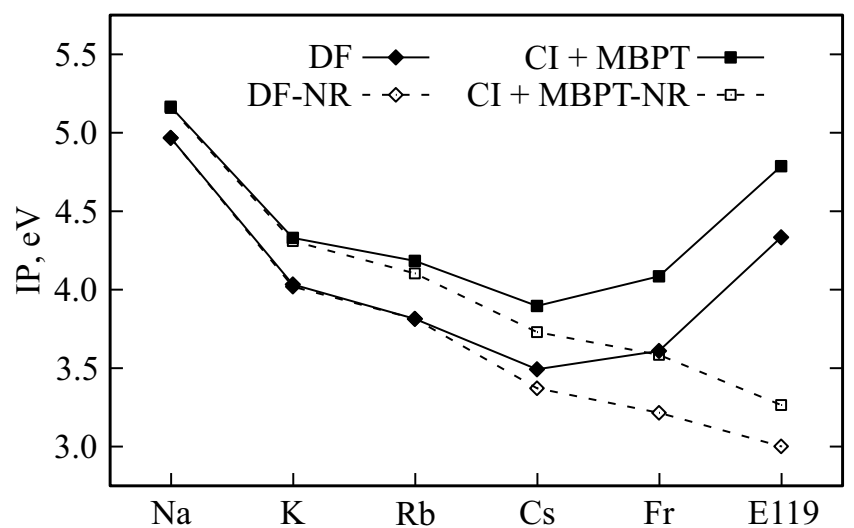

Рис. 3. Потенциалы ионизации (IP) элементов первой группы таблицы Менделеева (в eV). DF - метод Дирака-Фока, $\mathrm{CI}+\mathrm{MBPT}$ - метод наложения конфигураций в сочетании $\mathrm{c}$ теорией возмущений; NR - нерелятивистский предел. 


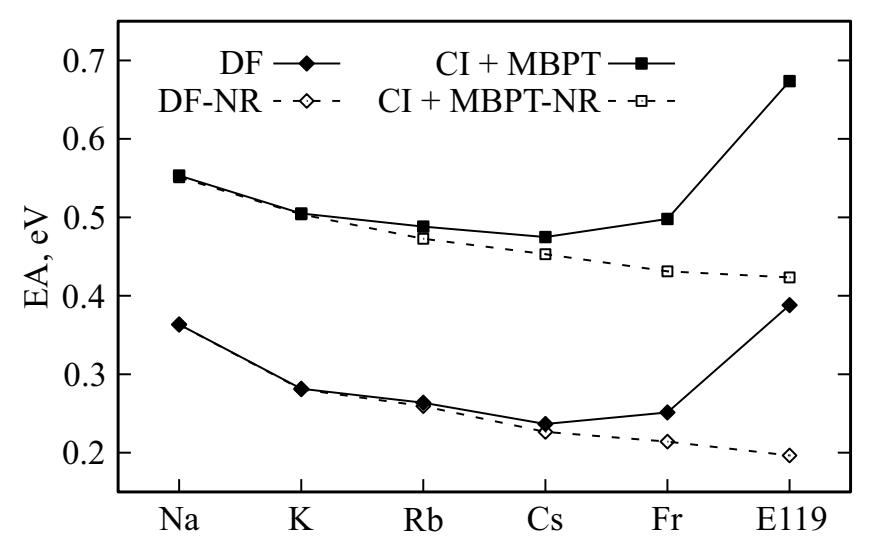

Рис. 4. Значения сродства к электрону (ЕА) элементов первой группы таблицы Менделеева (в eV). Обозначения такие же, как на рис. 3.

Сравнивая значения, полученные методами DF и CI+MВРТ, можно судить о вкладе корреляционных эффектов в величины IP и ЕА. Для потенциалов ионизации энергия корреляции составляет величину порядка $0.3-0.4 \mathrm{eV}$ для всех элементов группы щелочных элементов, представленных в табл. 2, кроме $\mathrm{Na}(Z=11)$, для которого она равна примерно $0.2 \mathrm{eV}$. Вклад энергии корреляции в сродство к электрону лежит в интервале $0.2-0.3 \mathrm{eV}$, и он монотонно возрастает с ростом $Z$.

В табл. 4 представлены результаты наших расчетов КЭД поправок к потенциалу ионизации. Как видно из таблицы, наши данные, полученные методами DF и CI+MBРТ с использованием модельного КЭД потенциала (QEDMOD) [26], мало отличаются друг от друга. Для сравнения в четвертой колонке (VRAD) приведены результаты, полученные нами методом DF с использованием радиационного потенциала [42]. В последней колонке представлены соответствующие результаты из работы [15].

В табл. 5 приведены значения потенциалов ионизации, полученные в данной работе методом CI+MBPT, с добавлением КЭД вкладов. В этой же таблице представлены результаты расчетов IP методом связанных кластеров [15] с учетом КЭД поправок, а также доступные экспериментальные значения. Как видно из сравнения, наши данные хорошо согласуются с результатами работы [15] и с экспериментом в пределах погрешности наших вычислений, которую мы оцениваем как $0.1 \mathrm{eV}$. Такая оценка погрешности обусловлена относительно небольшим размером базиса и вкладом трехкратных возбуждений, которые не учитывались в данной работе.

Значения сродства к электрону без учета КЭД поправок, полученные в данной работе методом CI+MBPT, представлены в табл. 6. В этой же таблице приведены результаты расчетов методом связанных кластеров [16]. Здесь также наблюдается хорошее согласие наших данных с результатами работы [16] и с экспериментом. Погрешность расчетов ЕА мы также оцениваем как $0.1 \mathrm{eV}$.
Таблица 4. КЭД вклад в потенциал ионизации элементов первой группы таблицы Менделеева (в meV). QEDMOD модельный оператор, VRAD - радиационный потенциал; DF - метод Дирака-Фока, CI+MBPT - метод наложения конфигураций в сочетании с теорией возмущений

\begin{tabular}{r|c|c|c|c}
\hline \multirow{2}{*}{$Z$} & \multicolumn{2}{|c|}{ QEDMOD } & \multirow{2}{*}{ VRAD } & \multirow{2}{*}{ Работа } \\
\cline { 2 - 4 } & DF & CI+MBPT & DF & {$[15]$} \\
\hline 11 & -0.3 & -0.3 & -0.3 & \\
19 & -0.5 & -0.6 & -0.5 & \\
37 & -1.3 & -1.5 & -1.3 & -1.3 \\
55 & -2.0 & -2.3 & -2.0 & -2.2 \\
87 & -4.8 & -5.1 & -4.7 & -3.6 \\
119 & -12.3 & -12.6 & -9.8 & -10.3
\end{tabular}

Таблица 5. Потенциалы ионизации элементов первой группы таблицы Менделеева (в еV). Сравнение с экспериментальными данными и результатами работы [15]

\begin{tabular}{r|c|c|c}
\hline$Z$ & Данная работа & Работа $[15]$ & Эксперимент \\
\hline 11 & 5.159 & & 5.139 \\
19 & 4.323 & & 4.341 \\
37 & 4.175 & 4.181 & 4.177 \\
55 & 3.887 & 3.901 & 3.894 \\
87 & 4.074 & 4.079 & 4.073 \\
119 & 4.768 & 4.783 &
\end{tabular}

Примечание. Экспериментальные данные взяты из работ $[43,44]$ для $\mathrm{Na} ;[45,46]$ для $\mathrm{K} ;[46,47]$ для $\mathrm{Rb} ;[48]$ для $\mathrm{Cs} ;[49]$ для $\mathrm{Fr}$.

Таблица 6. Значения сродства к электрону для элементов первой группы таблицы Менделеева (в eV). Сравнение с экспериментальными данными и результатами работы [16]

\begin{tabular}{r|c|c|c}
\hline$Z$ & Данная работа & Работа $[16]$ & Эксперимент \\
\hline 11 & 0.553 & 0.548 & 0.548 \\
19 & 0.505 & 0.503 & 0.501 \\
37 & 0.488 & 0.486 & 0.486 \\
55 & 0.475 & 0.471 & 0.472 \\
87 & 0.498 & 0.486 & \\
119 & 0.674 & 0.649 &
\end{tabular}

Примечание. Экспериментальные данные взяты из работ [50] для Na; $[51,52]$ для $\mathrm{K} ;[53]$ для $\mathrm{Rb} ;[51,54]$ для $\mathrm{Cs}$.

\section{4. Заключение}

В данной работе были рассмотрены химические характеристики сверхтяжелого элемента эка-франция $(Z=119)$ и его более легких гомологов. Были выполнены расчеты среднеквадратичных радиусов и среднеквадратичных отклонений валентной $n s$-оболочки, которые характеризуют степень локализации валентной электронной плотности. Полученные результаты свидетельствуют о том, что структура валентных оболочек сохраняется при переходе от более легких гомологов к эка-францию. Это говорит о том, что он, как и следовало 
ожидать, относится к первой группе элементов таблицы Менделеева.

Нами также были получены нерелятивистские и релятивистские значения потенциалов ионизации и сродства к электрону для эка-франция и его гомологов. Было установлено, что релятивистские эффекты нарушают монотонный характер уменьшения IP и ЕА, который имеет место в нерелятивистском пределе. Этот факт объясняется эффектом контрактации $s$ - и $p$-оболочек при учете релятивистских поправок и согласуется с аналогичным, ранее сформулированным утверждением [11].

В дальнейшем мы планируем расширить представленные здесь расчеты с использованием метода CI+MBPT на целый ряд сверхтяжелых элементов с $Z=118-130$.

\section{Финансирование работы}

Исследование выполнено при финансовой поддержке РФФИ и Госкорпорации „Росатом“ в рамках научного проекта № 20-21-00098.

\section{Конфликт интересов}

Авторы заявляют, что у них нет конфликта интересов.

\section{Список литературы}

[1] Oganessian Y.T., Dmitriev S.N. // Russ. Chem. Rev. 2016. V. 85. P. 901.

[2] Oganessian Y. // Nucl. Phys. News. 2019. V. 29. P. 5.

[3] Nazarewicz W. // Nature Phys. 2018. V. 14. P. 537.

[4] Theoretical Chemistry and Physics of Heavy and Superheavy Elements. Progress in Theoretical Chemistry and Physics. V. 11 / Ed. by Kaldor U., Wilson S. Springer Netherlands, 2003. XIX $+565 \mathrm{p}$.

[5] The chemistry of superheavy elements / Ed. by Schädel M., Shaughnessy D. Berlin Heidelberg: Springer-Verlag, 2014. XIII $+521 \mathrm{p}$.

[6] Eliav E., Fritzsche S., Kaldor U. // Nucl. Phys. A. 2015. V. 944. P. 518.

[7] Schwerdtfeger P., Pašteka L.F., Punnett A., Bowman P.O. // Nucl. Phys. A. 2015. V. 944. P. 551.

[8] Jerabek P., Schuetrumpf B., Schwerdtfeger P., Nazarewicz $W$. // Phys. Rev. Lett. 2018. V. 120. P. 053001.

[9] Lackenby B.G.C., Dzuba V.A., Flambaum V.V. // Phys. Rev. A. 2018. V. 98. P. 042512.

[10] Pershina V. // Radiochim. Acta. 2019. V. 107. P. 833.

[11] Eliav E., Borschevsky A., Kaldor U. // Nucl. Phys. News. 2019. V. 29. P. 16.

[12] Kaygorodov M.Y., Kozhedub Y.S., Tupitsyn I.I., Shabaev V.M. // PoS(FFK2019). 2020. V. 353. P. 036.

[13] Nefedov V.I., Trzhaskovskaya M.B., Yarzhemskii V.G. // Dok1. Phys. Chem. 2006. V. 408. P. 149.

[14] Landau A., Eliav E., Ishikawa Y., Kaldor U. // J. Chem. Phys. 2001. V. 115. P. 2389.

[15] Eliav E., Vilkas M.J., Ishikawa Y., Kaldor U. // Chem. Phys. 2005. V. 311. P. 163.

[16] Eliav E., Vilkas M.J., Ishikawa Y., Kaldor U. // J. Chem. Phys. 2005. V. 122. P. 224113.
[17] Lim I.S., Schwerdtfeger P., Metz B., Stoll H. // J. Chem. Phys. 2005. V. 122. P. 104103.

[18] Borschevsky A., Pershina V., Eliav E., Kaldor U. // J. Chem. Phys. 2013. V. 138. P. 124302.

[19] Sucher J. // Phys. Rev. A. 1980. V. 22. P. 348.

[20] Mittleman M.H. // Phys. Rev. A. 1981. V. 24. P. 1167.

[21] Angeli I., Marinova K.P. // At. Data Nucl. Data Tables. 2013. V. 99. P. 69.

[22] Братцев В.Ф., Дейнека Г.Б., Тупищын И.И. // Изв. АН CССР: Сер. физ. 1977. Т. 41. С. 2655.

[23] Tupitsyn I.I., Shabaev V.M., Crespo López-Urrutia J.R., Draganić I., Soria Orts R., Ullrich J. // Phys. Rev. A. 2003. V. 68. P. 022511.

[24] Tupitsyn I.I., Volotka A.V., Glazov D.A., Shabaev V.M., Plunien G., Crespo López-Urrutia J.R., Lapierre A., Ullrich J. // Phys. Rev. A. 2005. V. 72. P. 062503.

[25] Olsen J., Roos B.O., Jørgensen P., Jensen H.J.A. // J. Chem. Phys. 1988. V. 89. P. 2185.

[26] Shabaev V.M., Tupitsyn I.I., Yerokhin V.A. // Phys. Rev. A. 2013. V. 88. P. 012513.

[27] Tupitsyn I.I., Kozlov M.G., Safronova M.S., Shabaev V.M., Dzuba V.A. // Phys. Rev. Lett. 2016. V. 117. P. 253001.

[28] Pašteka L.F., Eliav E., Borschevsky A., Kaldor U., Schwerdtfeger P. // Phys. Rev. Lett. 2017. V. 118. P. 023002.

[29] Machado J., Szabo C.I., Santos J.P., Amaro P., Guerra M., Gumberidze A., Guojie Bian, Isac J.M., Indelicato P. // Phys. Rev. A. 2018. V. 97. P. 032517.

[30] Zaytsev V.A., Maltsev I.A., Tupitsyn I.I., Shabaev V.M. // Phys. Rev. A. 2019. V. 100. P. 052504.

[31] Kumar R., Chattopadhyay S., Mani B.K., Angom D. // Phys. Rev. A. 2020. V. 101. P. 012503.

[32] Shabaev V.M., Tupitsyn I.I., Kaygorodov M.Y., Kozhedub Y.S., Malyshev A.V., Mironova D.V. // Phys. Rev. A. 2020. V. 101. P. 052502.

[33] Serber R. // Phys. Rev. 1935. V. 48. P. 49.

[34] Uehling E.A. // Phys. Rev. 1935. V. 48. P. 55.

[35] Fullerton L.W., Rinker Jr. G.A. // Phys. Rev. A. 1976. V. 13. P. 1283.

[36] Soff G., Mohr P.J. // Phys. Rev. A. 1988. V. 38. P. 5066.

[37] Manakov N.L., Nekipelov A.A., Fainshtein A.G. // Sov. Phys. JETP. 1989. V. 68. P. 673.

[38] Persson H., Lindgren I., Salomonson S., Sunnergren P. // Phys. Rev. A. 1993. V. 48. P. 2772.

[39] Fainshtein A.G., Manakov N.L., Nekipelov A.A. // J. Phys. B: At. Mol. Opt. Phys. 1991. V. 24. P. 559.

[40] Shabaev V.M., Tupitsyn I.I., Yerokhin V.A. // Comp. Phys. Commun. 2015. V. 189. P. 175.

[41] Shabaev V.M., Tupitsyn I.I., Yerokhin V.A. // Comp. Phys. Commun. 2018. V. 223. P. 69.

[42] Flambaum V.V., Ginges J.S.M. // Phys. Rev. A. 2005. V. 72. P. 052115.

[43] Ciocca M., Burkhardt C.E., Leventhal J.J., Bergeman T. // Phys. Rev. A. 1992. V. 45. P. 4720.

[44] Baugh J.F., Burkhardt C.E., Leventhal J.J., Bergeman T. // Phys. Rev. A. 1998. V. 58. P. 1585.

[45] Lorenzen C.J., Niemax K., Pendrill L.R. // Opt. Commun. 1981. V. 39. P. 370.

[46] Lorenzen C.J., Niemax K. // Phys. Scr. 1983. V. 27. P. 300.

[47] Johansson I. // Ark. Fys. 1961. V. 20. P. 135.

[48] Deiglmayr J., Herburger H., Saßmannshausen H., Jansen P., Schmutz H., Merkt F. // Phys. Rev. A. 2016. V. 93. P. 013424. 
[49] Arnold E., Borchers W., Duong H.T., Juncar P., Lerme J., Lievens P., Neu W., Neugart R., Pellarin M., Pinard J., Vialle J.L., Wendt K., ISOLDE // J. Phys. B: At. Mol. Opt. Phys. 1990. V. 23. P. 3511.

[50] Hotop H., Lineberger W.C. // J. Phys. Chem. Ref. Data. 1985. V. 14. P. 731.

[51] Slater J., Read F.H., Novick S.E., Lineberger W.C. // Phys. Rev. A. 1978. V. 17. P. 201.

[52] Andersson K.T., Sandström J., Kiyan I.Y., Hanstorp D., Pegg D.J. // Phys. Rev. A. 2000. V. 62. P. 022503.

[53] Frey P., Breyer F., Holop H. // J. Phys. B: Atom. Mol. Phys. 1978. V. 11. P. L589.

[54] Scheer M., Thøgersen J., Bilodeau R.C., Brodie C.A., Haugen H.K., Andersen H.H., Kristensen P., Andersen T. // Phys. Rev. Lett. 1998. V. 80. P. 684. 